\title{
PERIODIC HOMOGENIZATION OF THE INVISCID G-EQUATION FOR INCOMPRESSIBLE FLOWS*
}

\author{
JACK XIN $^{\dagger}$ AND YIFENG YU
}

\begin{abstract}
G-equations are popular front propagation models in combustion literature and describe the front motion law of normal velocity equal to a constant plus the normal projection of fluid velocity. G-equations are Hamilton-Jacobi equations with convex but non-coercive Hamiltonians. We prove homogenization of the inviscid G-equation for space periodic incompressible flows. This extends a two space dimensional result in [26]. We construct approximate correctors to bypass the lack of compactness due to the non-coercive Hamiltonian. The existence of approximate correctors rely on a local reachability property of the controlled flow trajectory as well as incompressibility of the flow. Homogenization then follows from the comparison principle and the perturbed test function method. The effective Hamiltonian is convex and homogeneous of degree one. It is also coercive if we further assume that the flow is mean zero.
\end{abstract}

Key words. Non-coercive Hamilton-Jacobi equations, controlled flow, local reachability, incompressibility, periodic homogenization.

AMS subject classifications. 70H20, 76M50, 76M45, 76N20.

\section{Introduction}

Front or interface propagation in fluid flows is a robust nonlinear phenomenon arising in liquid phase chemical reaction, and premixed flame propagation in fluid turbulence $[13,33,32]$ among other applications. Mathematical models range from reaction-diffusion-advection equations to advective Hamilton-Jacobi equations (HJ), $[10,14,17,28,39,40,41]$. A particular HJ equation, the so called G-equation, is quite popular in the combustion science literature $[27,36,42,16]$. The inviscid G-equation is

$$
G_{t}+V(x) \cdot D_{x} G=s_{l}\left|D_{x} G\right|
$$

where $G$ is a scalar function (the level set function of the interface), $x \in \mathbb{R}^{n}, V(x)$ : $\mathbb{R}^{n} \rightarrow \mathbb{R}^{n}$ is a prescribed flow velocity field, $s_{l}$ is a positive constant (laminar front speed), and $D_{x}$ is the spatial gradient operator. For simplicity, we only consider time independent flows in this paper. The G-equation (1.1) is the level set equation of the interface motion law: the exterior normal velocity of the interface equals the laminar speed $s_{l}$ plus the projection of the fluid velocity along the normal; see [30, Chapter $6]$ and [31].

A fundamental problem in turbulent combustion is to study the large time front speed, or the asymptotic growth rate $\lim _{t \rightarrow+\infty} G(x, t) / t$, and analyze its dependence on the advection field $V$. Such a limit (if it exists) is called the turbulent front speed, $[32,41]$. The large time front speed may be captured by first performing a scaling transform $G^{\epsilon}(x, t)=\epsilon G(x / \epsilon, t / \epsilon)$, then taking the limit $\epsilon \rightarrow 0$. The transformed equation is:

$$
G_{t}^{\epsilon}+V(x / \epsilon) \cdot D_{x} G^{\epsilon}=s_{l}\left|D_{x} G^{\epsilon}\right|
$$

\footnotetext{
*Received: December 15, 2009; accepted: March 14, 2010. Communicated by Weinan E.

${ }^{\dagger}$ Department of Mathematics, University of California at Irvine, Irvine, CA 92697, USA (jack.xin@uci.edu).

${ }^{\ddagger}$ Department of Mathematics, University of California at Irvine, Irvine, CA 92697, USA (yifengy@uci.edu).
} 
which is the homogenization problem of Hamilton-Jacobi equation. Here $\epsilon \rightarrow 0$ plays the role of $t \rightarrow+\infty$. Homogenization of Hamilton-Jacobi (HJ) equation

$$
u_{t}^{\epsilon}+H\left(\frac{x}{\epsilon}, D_{x} u^{\epsilon}\right)=0,
$$

when the Hamiltonian $H=H(x, p)$ is a 1-periodic function in $x$ (so called periodic homogenization) was originated in [24] in the 1980's, and further developed [18, 19] to include viscous HJs and fully nonlinear equations. Besides periodicity of $H$ in $x$, the Hamiltonian is required to be coercive $[24,18,19]$ :

$$
|H(x, p)| \rightarrow+\infty \text {, as }|p| \rightarrow+\infty \text {, uniformly in } x .
$$

Recently, much progress has been made in extending homogenization to stationary ergodic media for convex and coercive inviscid and viscous HJs [37, 34, 35, 25, 22, 23, $38]$.

The Hamiltonian of the G-equation is $H(x, p)=-s_{l}|P|+V(x) \cdot P$, which is not coercive if $V$ has large enough amplitude as in a strong advection regime. In this paper, we are concerned with homogenization of the inviscid G-equation (1.2) for periodic incompressible flow $V$ (i.e., $\operatorname{div}(V)=\nabla \cdot V=0$ ). The key step is to find approximate correctors since exact correctors may not exist in case of the non-coercive Hamiltonian. We first use the incompressibility to derive the existence of one-sided approximate correctors. Then the other side follows from suitable reachability properties of the flow under control. When $n=2$, a simple argument shows that any point can reach the region where $|V|$ is small through a controlled flow within finite time [26]. This however is no longer true in higher dimensions. Instead, we discover that a local reachability is sufficient. We establish it first, then apply it to the existence of an effective Hamiltonian by a covering argument. The convergence of time dependent solutions follows by the comparison principle $[9,21]$ and the perturbed test function method [19]. We further show that the effective Hamiltonian in incompressible flow is convex, homogeneous of degree one and coercive (if the mean of $V$ is also zero). Though homogenization of non-coercive HJs have been studied before [5, 7, 11], the results do not apply to the G-equation (1.1). Likewise, a property called "uniform exact controllability" (any two points in space can be connected by a controlled flow trajectory within finite time) and its implication for homogenization are investigated before $[2,4,6]$. However such a controllability is difficult to verify for the G-equation (1.1). The local reachability defined in this paper is much weaker and holds for any continuous flow.

The homogenization of the viscous G-equation (including $\kappa \Delta G$ on the right hand side of (1.1), $\kappa>0$ ) was studied recently [26]. In this case, the cell (corrector) problem has exact classical solutions. Qualitatively, the viscous Hamiltonian is the same as the inviscid effective Hamiltonian. However, they are very different quantitatively. An interesting question is how the effective Hamiltonian depends on viscosity $\kappa$ and the flow patterns in various asymptotic regimes. A comparison study was carried out recently [29] in terms of bounds and scaling behavior of the effective Hamiltonians for shear flow, gradient flow, and cellular flow.

The paper is organized as follows. In section 2, we define and establish the local reachability property of the controlled flow trajectories, then use this to prove the existence of approximate correctors as well as qualitative properties of the effective Hamiltonians. In section 3, we prove the convergence of homogenization limit for time dependent solutions of the inviscid G-equation (1.1). In section 4, we conclude with remarks on future work. 
After this work was completed, we learned that, by a different approach, Nolen, Cardaliaguet, and Souganidis [12] proved periodic homogenization of (1.2) when $\int_{\mathbb{T}^{n}}|\nabla \cdot V|^{n} d x$ is small ( $\mathbb{T}^{n}$ the $\mathrm{n}$-dimensional unit torus). Our main result extends to $V=V_{1}+V_{2}$, where $\operatorname{div}\left(V_{1}\right)=0, \max _{\mathbb{T}^{n}}\left|V_{2}\right|<s_{l}$.

\section{Existence of approximate correctors}

Let us set $s_{l}=1$ with no loss of generality, and consider a 1-periodic divergence free Lipschitz continuous vector field $V=V(x), u=-G$. Then (1.2) becomes

$$
u_{\epsilon, t}+V\left(\frac{x}{\epsilon}\right) \cdot D u_{\epsilon}+\left|D u_{\epsilon}\right|=0 .
$$

The formal two-scale homogenization ansatz,

$$
u_{\epsilon}(x, t)=u_{0}(x, t)+\epsilon u_{1}\left(x, t, \frac{x}{\epsilon}\right)+\ldots,
$$

gives to leading order,

$$
u_{0, t}+V(y) \cdot\left(D_{x} u_{0}+D_{y} u_{1}\right)+\left|D_{x} u_{0}+D_{y} u_{1}\right|=0 .
$$

The standard cell (corrector) problem is: given any vector $P \in \mathbb{R}^{n}$, find a unique number $\bar{H}(P)$ such that the equation

$$
\left|P+D_{y} u\right|+\left(P+D_{y} u\right) \cdot V(y)=\bar{H}(P), \quad y \in \mathbb{T}^{n}
$$

has a periodic solution $u=u(y)$ on $\mathbb{T}^{n}$. If the cell problem is solvable, $u_{0}$ then formally satisfies the homogenized Hamilton-Jacobi equation:

$$
u_{0, t}+\bar{H}\left(D_{x} u_{0}\right)=0
$$

However, exact solutions of cell problem may not exist due to the lack of coercivity of the inviscid G-equation. This is manifested as lack of gradient estimate in (2.4) where $\left|D_{y} u\right|$ is not bounded a-priori if the amplitude of the flow $V$ exceeds one, in contrast to the case of coercive Hamilton-Jacobi equation (if $\left|P+D_{y} u\right|^{1+\eta}$ replaces $\left.\left|P+D_{y} u\right|, \eta>0\right)$.

We shall prove in this section that the cell problem has an approximate solution by combined use of the local reachability property of the controlled flow trajectory and the incompressibility. Let us begin with

Definition 2.1 (Controlled Flow Trajectory). A controlled flow trajectory associated to $V$ is $\xi \in W^{1, \infty}\left([0, T] ; \mathbb{R}^{n}\right)$ such that for a.e. $t \in(0, T)$,

$$
\dot{\xi}(t)=\alpha(t)+V(\xi(t))
$$

where the control $\alpha(t) \in L^{\infty}\left([0, T] ; B_{1}(0)\right)$.

Definition 2.2 (Reachability). Suppose that $E$ and $F$ are two subsets of $\mathbb{R}^{n}$. We say that $F$ can be fully reached by $E$ within time $T$ if for any $x \in E$ and $y \in F$ there exists a controlled flow trajectory $\xi_{x, y}:[0, \hat{T}] \rightarrow \mathbb{R}^{n}$ such that $\xi_{x, y}(0)=x, \xi_{x, y}(\hat{T})=y$ and $\hat{T} \leq T$.

Lemma 2.1 (Local Reachability). For any $x \in \mathbb{R}^{n}$, there exists $x^{\prime} \in \mathbb{R}^{n}$, a positive number $r_{x}>0$ such that $B_{r_{x}}\left(x^{\prime}\right)$ can be fully reached by $B_{r_{x}}(x)$ within time less than or equal to 1. 
Proof. Fix $x \in \mathbb{R}^{n}$. Since $V$ is continuous, choose $r_{0}>0$ such that $\mid V(y)-$ $V(x) \mid<\frac{1}{2}$ for $y \in B_{r_{0}}(x)$. For $y \in B_{r_{0}}(x)$ and $\bar{\alpha} \in B_{\frac{1}{2}}(0)$, we can design proper control $\alpha(t)$ such that the corresponding controlled flow trajectory starting from $y$ is the straight line $\xi(t)=y+t(\bar{\alpha}+V(x))$ within $B_{r_{0}}(x)$. In fact, writing the flow as solution to the ODE

$$
\dot{\xi}=\bar{\alpha}+V(x)=\bar{\alpha}+V(x)-V(\xi(t))+V(\xi(t)),
$$

which is in the form of $(2.6)$ with control $\alpha(t)=\bar{\alpha}+V(x)-V(\xi(t))$, clearly $|\alpha| \leq 1$.

Now choose $t_{0} \in(0,1)$ such that

$$
x^{\prime}=x+t_{0} V(x) \in B \frac{r_{0}}{2}(x),
$$

and $r_{x}>0$ satisfying

$$
r_{x}<\frac{t_{0}}{4}
$$

Now for $y \in B_{r_{x}}(x)$ and $y^{\prime} \in B_{r_{x}}\left(x^{\prime}\right)$, there exists $\bar{\alpha} \in B_{\frac{1}{2}}(0)$ such that

$$
y^{\prime}=y+t_{0}(\bar{\alpha}+V(x)) .
$$

To see this, let

$$
\bar{\alpha}=\frac{y^{\prime}-y-t_{0} V(x)}{t_{0}}
$$

Then by (2.7) and (2.8),

$$
|\bar{\alpha}| \leq \frac{|y-x|+\left|y^{\prime}-x^{\prime}\right|}{t_{0}}<\frac{1}{2} .
$$

Hence $B_{r_{x}}(\hat{x})$ can be fully reached by $B_{r_{x}}(x)$ at time $t_{0}<1$. See figure 1 for an illustration of the controlled flow trajectories inside the ball $B_{r_{0}}(x)$, where $V(x) \neq 0$. Note that if $x$ happens to be an equilibrium point of $V(V(x)=0), x^{\prime}=x$.

Next we establish an inequality for a subsolution of the modified cell problem at two points connected by a controlled flow trajectory.

Lemma 2.2. Suppose that $u \in C\left(\mathbb{T}^{n}\right)$ is a viscosity subsolution of

$$
\lambda u+|P+D u|+V(x) \cdot(P+D u)=0 \quad \text { in } \mathbb{R}^{n} .
$$

Then for any controlled flow trajectory $\xi:[0, T] \rightarrow \mathbb{R}^{n}$,

$$
u(\xi(T))-e^{-\lambda T} u(\xi(0)) \leq-\int_{0}^{T} P \cdot \dot{\xi} e^{\lambda(s-T)} d s
$$

Proof. For $\delta>0$, consider the super involution

$$
u_{\delta}(x)=\sup _{y \in \mathbb{R}^{n}}\left(u(y)-\frac{1}{\delta}|x-y|^{2}\right)=\sup _{z \in \mathbb{R}^{n}}\left(u(x+z)-\frac{1}{\delta}|z|^{2}\right) .
$$




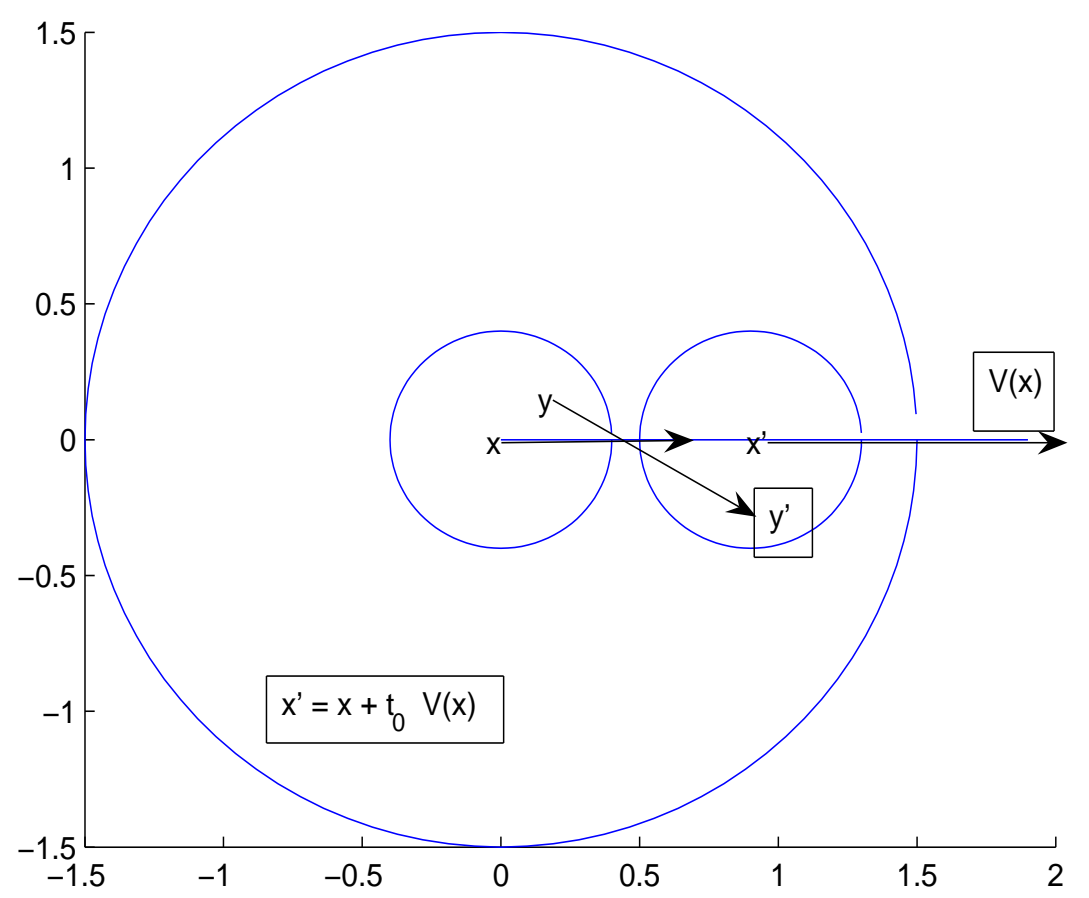

FIG. 2.1. Sketch of controlled flow trajectories in the ball $B_{r_{0}}(x)$ where $V(x) \neq 0$, the outer circle has radius equal to $r_{0}$, and the inner circles have radii equal to $r_{x}$. The controlled flow from $B_{r_{x}}(x)$ to $B_{r_{x}}\left(x^{\prime}\right)$ is generic for $V$.

It is clear that $u_{\delta}$ is periodic, semiconvex, and Lipschitz continuous. Since $u$ is bounded,

$$
u_{\delta}(x)=\sup _{z \in B_{\tilde{C} \sqrt{\delta}}(0)}\left(u(x+z)-\frac{1}{\delta}|z|^{2}\right),
$$

where $\tilde{C}=\sqrt{2 \max _{\mathbb{T}^{n}}|u|}$. Hence $u_{\delta}$ is a viscosity subsolution of

$$
\lambda u_{\delta}+\left(1-C_{u} \sqrt{\delta}\right)\left|P+D u_{\delta}\right|+V(x) \cdot\left(P+D u_{\delta}\right) \leq o(1)
$$

for some constant $C_{u}$ which is independent of $\delta$ and $\lim _{\delta \rightarrow 0} o(1)=0$. Since the Hamiltonian is convex in $P$, by mollifying $u_{\delta}$ we may assume that $u_{\delta}$ is $C^{1}$. Suppose that $\dot{\xi}(t)=\alpha(t)+V(\xi(t))$. Let $\xi_{\delta}:[0, T] \rightarrow \mathbb{R}^{n}$ be the control satisfying that

$$
\left\{\begin{array}{l}
\dot{\xi}_{\delta}=\left(1-C_{u} \sqrt{\delta}\right) \alpha+V\left(\xi_{\delta}\right) \\
\xi_{\delta}(0)=\xi(0) .
\end{array}\right.
$$

Then for a.e. $t$

$$
\begin{aligned}
\frac{d\left(P \cdot \xi_{\delta}+u_{\delta}\left(\xi_{\delta}\right)\right)}{d t} & =\left(P+D u_{\delta}\left(\xi_{\delta}\right)\right) \cdot\left(\left(1-C_{u} \sqrt{\delta}\right) \alpha(t)+V\left(\xi_{\delta}(t)\right)\right) \\
& \leq\left(1-C_{u} \sqrt{\delta}\right)\left|P+D u_{\delta}\left(\xi_{\delta}\right)\right|+V\left(\xi_{\delta}\right)\left(P+D u_{\delta}\left(\xi_{\delta}\right)\right) \\
& \leq-\lambda u_{\delta}\left(\xi_{\delta}\right)+o(1) .
\end{aligned}
$$


So

$$
\frac{d}{d t}\left(e^{\lambda t} u_{\delta}\left(\xi_{\delta}(t)\right)\right) \leq-e^{\lambda t} P \cdot \dot{\xi}_{\delta}(t)+o(1)
$$

Therefore

$$
u_{\delta}\left(\xi_{\delta}(T)\right)-e^{-\lambda T} u\left(\xi_{\delta}(0)\right) \leq-\int_{0}^{T} P \cdot \dot{\xi}_{\delta} e^{\lambda(s-T)} d s+o(1) .
$$

Sending $\delta \rightarrow 0$, the above lemma holds.

The standard Perron's method implies that for given $\lambda>0$, there exists a unique periodic viscosity solution $u_{\lambda} \in C\left(\mathbb{T}^{n}\right)$ of

$$
\lambda u_{\lambda}+\left|P+D u_{\lambda}\right|+V(x) \cdot\left(P+D u_{\lambda}\right)=0 \quad \text { in } \mathbb{R}^{n} .
$$

We refer to [15] for details. By the maximum principle,

$$
\left|\lambda u_{\lambda}\right| \leq|P|\left(1+\max _{\mathbb{T}^{n}}|V|\right) .
$$

Hereafter we assume that $V$ is an incompressible vector field, i.e., $\nabla \cdot V=0$. The following is the key lemma of this section.

Lemma 2.3. For any sequence $\lambda_{m} \rightarrow 0$ as $m \rightarrow+\infty$, there exists a subsequence $\lambda_{m_{k}} \rightarrow 0$ as $k \rightarrow+\infty$ such that

$$
\lim _{k \rightarrow+\infty} \lambda_{m_{k}} u_{\lambda_{m_{k}}}=c \quad \text { uniformly in } \mathbb{R}^{n}
$$

for some constant $c \in \mathbb{R}$.

Proof. Since $\mathbb{T}^{n}$ is compact, there exist finite points $\left\{x_{i}\right\}_{i=1}^{(l)}$ such that

$$
\mathbb{T}^{n} \subset \cup_{i=1}^{(l)} B_{\frac{r_{x_{i}}}{2}}\left(x_{i}\right),
$$

where $r_{x_{i}}$ is from Lemma 2.1 corresponding to $x_{i}$.

Step I: Note that $v_{m}=\lambda_{m} u_{\lambda_{m}}$ is a viscosity solution of

$$
\lambda_{m} v_{m}+\left|\lambda_{m} P+D v_{m}\right|+V(x) \cdot\left(\lambda_{m} P+D v_{m}\right)=0 .
$$

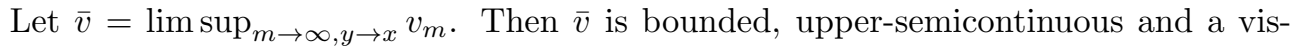
cosity subsolution of

$$
|D \bar{v}|+V(x) \cdot D \bar{v} \leq 0 \quad \text { in } \mathbb{R}^{n} .
$$

As in the proof of Lemma 2.2, we consider the super involution $\bar{v}_{\delta}$ of $\bar{v}$. Then when $\delta$ is small enough, $\bar{v}_{\delta}$ is a viscosity subsolution of

$$
\frac{1}{2}\left|D \bar{v}_{\delta}\right|+V(x) \cdot D \bar{v}_{\delta} \leq 0 \quad \text { in } \mathbb{R}^{n} .
$$

Note that there is no error term o(1) at the right hand side since the above equation does not involve the zeroth order term. Integrating over $\mathbb{T}^{2}$, we derive that

$$
\int_{\mathbb{T}^{2}}\left|D \bar{v}_{\delta}\right| d x=0 .
$$


Hence $\bar{v}_{\delta}(x) \equiv c_{\delta}$ for some constant $c_{\delta} \in \mathbb{R}$. Upon a subsequence if necessary, we may assume that $\lim _{\delta \rightarrow 0} c_{\delta}=c$. Since $\lim _{\delta \rightarrow 0} \bar{v}_{\delta}=\bar{v}$, we obtain that

$$
\bar{v}(x) \equiv c
$$

for some constant $c \in \mathbb{R}$.

Step II: We claim that there exists a subsequence $\left\{\lambda_{m}^{(1)}\right\}$ of $\left\{\lambda_{m}\right\}$ such that $\lambda_{m}^{(1)} \rightarrow 0$ as $m \rightarrow+\infty$ and

$$
\lim _{m \rightarrow+\infty} \lambda_{m}^{(1)} u_{\lambda_{m}^{(1)}}=c \quad \text { uniformly in } \overline{B_{\frac{r_{x_{1}}}{2}}\left(x_{1}\right)} .
$$

In fact, let $x_{1}^{\prime}$ be the reachable point from $x_{1}$ as stated in Lemma 2.1. According to (2.9), there exists $z_{m} \rightarrow x_{1}^{\prime}, \lambda_{m}^{(1)} \rightarrow 0$ as $m \rightarrow+\infty$ such that

$$
\lim _{m \rightarrow+\infty} \lambda_{m}^{(1)} u_{\lambda_{m}^{(1)}}\left(z_{m}\right)=c .
$$

Fix $y \in B_{r_{x_{1}}}\left(x_{1}\right)$. By Lemma 2.1 for each $m$ there exists a controlled flow trajectory $\xi_{m}:\left[0, T_{m}\right] \rightarrow \mathbb{R}^{n}$ such that $\xi_{m}(0)=y, \xi_{m}\left(T_{m}\right)=z_{m}$, and $T_{m} \leq 1$. Thanks to Lemma 2.2 ,

$$
\begin{aligned}
u_{\lambda_{m}^{(1)}}\left(z_{m}\right)-e^{-\lambda_{m}^{(1)} T_{m}} u_{\lambda_{m}^{(1)}}(y) & \leq-\int_{0}^{T_{m}} P \cdot \dot{\xi}_{m} e^{\lambda_{m}^{(1)}\left(s-T_{m}\right)} d s . \\
& \leq T_{m}|P|\left(1+\max _{\mathbb{T}^{n}}|V|\right) \\
& \leq|P|\left(1+\max _{\mathbb{T}^{n}}|V|\right) .
\end{aligned}
$$

Accordingly, for $w \in B_{r_{x_{1}}}\left(x_{1}\right)$,

$$
\liminf _{m \rightarrow+\infty, y \rightarrow w} \lambda_{m}^{(1)} u_{\lambda_{m}^{(1)}}(y) \geq c=\limsup _{m \rightarrow+\infty, y \rightarrow w} \lambda_{m} u_{\lambda_{m}}(y) \geq \limsup _{m \rightarrow+\infty, y \rightarrow w} \lambda_{m}^{(1)} u_{\lambda_{m}^{(1)}}(y) .
$$

Hence our claim holds.

Step III: Similar to the derivation of (2.9), $\lim _{\sup _{m \rightarrow+\infty, y \rightarrow x}} \lambda_{m}^{(1)} u_{\lambda_{m}^{(1)}}(y) \equiv$ constant in $\mathbb{R}^{n}$. Combining with step II,

$$
\limsup _{m \rightarrow+\infty, y \rightarrow x} \lambda_{m}^{(1)} u_{\lambda_{m}^{(1)}}(y)=c \quad \text { for all } x \in \mathbb{R}^{n} .
$$

Then apply step II to the sequence $\left\{\lambda_{m}^{(1)}\right\}_{m \geq 1}$. We conclude that there exists a subsequence $\left\{\lambda_{m}^{(2)}\right\}_{m \geq 1}$ of $\left\{\lambda_{m}^{(1)}\right\}_{m \geq 1}$ such that $\lambda_{m}^{(2)} \rightarrow 0$ as $m \rightarrow+\infty$ and

$$
\lim _{m \rightarrow+\infty} \lambda_{m}^{(2)} u_{\lambda_{m}^{(2)}}=c \quad \text { uniformly in } \overline{B_{\frac{r_{2}}{2}}\left(x_{2}\right)} .
$$

The lemma follows after we repeat this procedure $l$ times.

Lemma 2.4 .

$$
\lim _{\lambda \rightarrow 0} \lambda u_{\lambda}=-\bar{H}(P), \quad \text { uniformly in } \mathbb{R}^{n},
$$

where $\bar{H}(P)$ is a constant. As a function of $P, \bar{H}(P)$ is Lipschitz continuous, convex and homogeneous of degree one. If $\int_{\mathbb{T}^{n}} V d x=0$, then $\bar{H}(P)$ is also coercive. 
Proof. By Lemma 2.3, we find a subsequence $\lambda_{m} \rightarrow 0$ as $m \rightarrow+\infty$ such that

$$
\lim _{m \rightarrow+\infty} \lambda_{m} u_{\lambda_{m}}=c \quad \text { uniformly in } \mathbb{R}^{n}
$$

for some constant $c$. We show that

$$
\lim _{\lambda \rightarrow 0} \lambda u_{\lambda}=c, \quad \text { uniformly in } \mathbb{R}^{n} .
$$

If not, owing to Lemma 2.3, then there exists another subsequence $\lambda_{m}^{\prime} \rightarrow 0$ as $m \rightarrow$ $+\infty$ such that

$$
\lim _{m \rightarrow+\infty} \lambda_{m}^{\prime} u_{\lambda_{m}^{\prime}}=c^{\prime} \neq c \quad \text { uniformly in } \mathbb{R}^{n} .
$$

Without loss of generality, we assume that $c^{\prime}>c$. Choose $c^{\prime}>t_{2}>t_{1}>c$. Then when $m$ is sufficiently large, $u_{\lambda_{m}}$ is a viscosity subsolution of

$$
\left|P+D u_{\lambda_{m}}\right|+V(x) \cdot\left(P+D u_{\lambda_{m}}\right) \geq-t_{1}
$$

and $u_{\lambda_{m}^{\prime}}$ is a viscosity supersolution of

$$
\left|P+D u_{\lambda_{m}^{\prime}}\right|+V(x) \cdot\left(P+D u_{\lambda_{m}^{\prime}}\right) \leq-t_{2} .
$$

This is impossible if we consider the place where $u_{\lambda_{m}}-u_{\lambda_{m}^{\prime}}$ attains minimum via a double variable method [15]. Let us denote $\bar{H}(P)=-c$.

Next we prove that $\bar{H}(P)$ is Lipschitz continuous. In fact, fix $\lambda$, and let $u_{P}$ and $u_{Q}$ be unique periodic viscosity solutions of the following two equations respectively:

$$
\lambda u_{P}+\left|P+D u_{P}\right|+V(x) \cdot\left(P+D u_{P}\right)=0
$$

and

$$
\lambda u_{Q}+\left|Q+D u_{Q}\right|+V(x) \cdot\left(Q+D u_{Q}\right)=0 .
$$

Then it is clear that $\tilde{u}_{P}=u_{P}+\frac{|P-Q|\left(1+\max _{\mathbb{T}} n|V|\right)}{\lambda}$ is a viscosity supersolution of

$$
\lambda \tilde{u}_{P}+\left|Q+D \tilde{u}_{P}\right|+V(x) \cdot\left(Q+D \tilde{u}_{P}\right) \geq 0 .
$$

Hence the comparison principle implies that $\lambda u_{Q} \leq \lambda u_{P}+|P-Q|\left(1+\max _{\mathbb{T}^{n}}|V|\right)$. Sending $\lambda \rightarrow 0$, we obtain that

$$
|\bar{H}(P)-\bar{H}(Q)| \leq|P-Q|\left(1+\max _{\mathbb{T}^{n}}|V|\right) .
$$

Next we prove that $\bar{H}$ is convex. Using super involution as in the proof of Lemma 2.2 , it is not hard to prove that $\tilde{u}=\frac{u_{P}+u_{Q}}{2}$ is a viscosity subsolution of

$$
\lambda \tilde{u}+\left|\frac{P+Q}{2}+D \tilde{u}\right|+V(x) \cdot\left(\frac{P+Q}{2}+D \tilde{u}\right) \leq 0 .
$$

Hence comparison principle implies that

$$
\lambda \tilde{u} \leq \lambda u_{\frac{P+Q}{2}} .
$$

Convexity follows after sending $\lambda \rightarrow 0$.

Finally, it is clear that for $s>0, u_{s}=s u_{\lambda}$ is a viscosity solution of

$$
\lambda u_{s}+\left|s P+D u_{s}\right|+V(x) \cdot\left(s P+D u_{s}\right)=0 .
$$

Thus $\bar{H}(P)$ is homogeneous of positive degree one.

Letting $s=1$ in (2.10), integrating over $\mathbb{T}^{n}$, applying Jensen's inequality, and sending $\lambda \rightarrow 0$, one sees that $\bar{H}(P) \geq|P|$ if $\int_{\mathbb{T}^{n}} V d x=0$ and thus it is coercive. 


\section{Convergence and homogenization}

Consider the homogenization problem of the inviscid G-equation:

$$
\begin{aligned}
& u_{\epsilon, t}+V\left(\frac{x}{\epsilon}\right) \cdot D u_{\epsilon}+\left|D u_{\epsilon}\right|=0, \\
& u_{\epsilon}(x, 0)=g(x),
\end{aligned}
$$

where $V$ is periodic and Lipschitz continuous, $g(x)$ is uniformly continuous and grows at most linearly, and $|g(x)| \leq C_{1}|x|+C_{2}$ for two constants $C_{1}$ and $C_{2}$. Such initial data includes the affine function for initiating traveling fronts. For each $\epsilon>0$, there exists a unique viscosity solution $u_{\epsilon} \in C\left(\mathbb{R}^{n} \times[0,+\infty)\right)$ which grows at most linearly in $t$ and $x$. The existence and uniqueness of $u_{\epsilon}$ follow from Corollary 2.1 in [8]. The existence part can also be deduced from the optimal control formulation [20, 29]. Hereafter, $C$ denotes a constant which depends only on advection field $V$ and the initial data $g$. After we establish the existence of approximate correctors, the proof of homogenization is standard. For the reader's convenience, we present details here.

We first show a growth bound of $u_{\epsilon}$ uniformly in $\epsilon$. LEMMA 3.1.

$$
\left|u_{\epsilon}(x, t)\right| \leq C(|x|+t+1) .
$$

Proof. Let $v(x, t)=\sqrt{|x|^{2}+1}+C t$. Choose $C$ large enough such that

$$
\begin{gathered}
v_{t}+V\left(\frac{x}{\epsilon}\right) \cdot D v+|D v|>0, \\
(-v)_{t}+V\left(\frac{x}{\epsilon}\right) \cdot D(-v)+|D(-v)|<0
\end{gathered}
$$

and

$$
C v(x, 0)=C \sqrt{|x|^{2}+1} \geq|g(x)| .
$$

Then the comparison principle (see Theorem 2.1 in [8]) implies that

$$
\left|u_{\epsilon}\right| \leq C v(x, t) \text {. }
$$

The following is our main result.

THEOREM 3.1. (Homogenization) Suppose that $V=V(x)$ is a Lipschitz continuous incompressible vector field (i.e., $\nabla \cdot V=0$ ). Then as $\epsilon \rightarrow 0$, $u_{\epsilon}$ locally uniformly converges to $u$ which grows at most linearly in $(x, t)$ and is the unique viscosity solution of

$$
\begin{aligned}
& u_{t}+\bar{H}(D u)=0, \\
& u(x, 0)=g,
\end{aligned}
$$

where $\bar{H}$ is the convex function on $\mathbb{R}^{n}$ given by Lemma 2.3.

In preparation for proving the above theorem, we first prove several lemmas. Since $g$ is uniformly continuous, for any $\tau>0$, there exists a $\delta>0$ such that

$$
|g(x)-g(y)| \leq \tau \quad \text { if }|x-y| \leq \delta
$$


For $x_{0} \in \mathbb{R}^{n}$, let $w_{x_{0}, \delta} \in C^{\infty}\left(\mathbb{R}^{n}\right)$ be a nonnegative function satisfying

(i) $w_{x_{0}, \delta}(x)=0$, for $\left|x-x_{0}\right| \leq \frac{\delta}{2}$,

(ii) $w_{x_{0}, \delta}(x)=\sqrt{|x|^{2}+1}$ for $\left|x-x_{0}\right| \geq \delta$.

Then we have:

Lemma 3.2. For any $x_{0} \in \mathbb{R}^{n}$, and $\epsilon \in(0,1)$,

$$
g\left(x_{0}\right)-\tau-\hat{C} w_{x_{0}, \delta}(x)-\hat{C} t \leq u_{\epsilon}(x, t) \leq g\left(x_{0}\right)+\tau+\hat{C} w_{x_{0}, \delta}(x)+\hat{C} t,
$$

where $\hat{C}$ is a constant depending on $C_{1}, C_{2}, V, w_{x_{0}, \delta}$ and $x_{0}$.

Proof. We just prove the " $\leq$ ". The other part is similar. Denote

$$
v(x, t)=g\left(x_{0}\right)+\tau+\hat{C} w_{x_{0}, \delta}(x)+\hat{C} t .
$$

It is clear that when $\hat{C}$ is sufficiently large

$$
u_{\epsilon}(x, 0)=g(x) \leq v(x, 0)
$$

and

$$
v_{t}+V\left(\frac{x}{\epsilon}\right) \cdot D v+|D v|>1
$$

Then the above lemma follows from Theorem 2.1 in [8].

The compactness of $\left\{u_{\epsilon}\right\}_{\epsilon>0}$ is a delicate issue. It is hard to prove directly that $\left\{u_{\epsilon}\right\}_{\epsilon>0}$ is equicontinuous. Instead we utilize techniques in [9] and [21] to bypass the lack of compactness. Let $U S C\left(\mathbb{R}^{n} \times[0, \infty)\right)\left(L S C\left(\mathbb{R}^{n} \times[0, \infty)\right)\right)$ denote the set of upper(lower) semicontinuous functions on $\mathbb{R}^{n} \times[0,+\infty)$. Let

and

$$
u^{*}(x, t)=\limsup _{\epsilon \rightarrow 0, y \rightarrow x, s \rightarrow t} u_{\epsilon}(y, s)
$$

$$
u_{*}(x, t)=\liminf _{\epsilon \rightarrow 0, y \rightarrow x, s \rightarrow t} u_{\epsilon}(y, s) .
$$

Clearly, $u^{*} \in U S C\left(\mathbb{R}^{n} \times[0,+\infty)\right)$ and $u_{*} \in L S C\left(\mathbb{R}^{n} \times[0,+\infty)\right)$. According to Lemma 3.2,

$$
u^{*}(x, 0)=u_{*}(x, 0)=g(x) .
$$

According to Lemma 2.3, we can find approximate viscosity solution of the cell problem, i.e., for any $\tau>0$, there exists a viscosity solution $u_{\tau} \in C\left(\mathbb{T}^{n}\right)$ of

$$
\bar{H}(P)-\tau \leq\left|P+D u_{\tau}\right|+V(x) \cdot\left(P+D u_{\tau}\right) \leq \bar{H}(P)+\tau .
$$

Then by perturbed test function methods [19], we have

Lemma 3.3. $u^{*}\left(u_{*}\right)$ is a viscosity subsolution (supersolution) of

$$
u_{t}+\bar{H}(D u)=0, \quad u(x, 0)=g(x) .
$$

\section{Proof of Theorem 3.1.}

Proof. Clearly, $u^{*}$ and $u_{*}$ satisfy the same growth estimates in Lemma 3.1. Since $\bar{H}$ is uniformly Lipschitz continuous, it follows from Theorem 2.1 (comparison principle) in [8],

$$
u^{*} \leq u_{*}
$$

On the other hand, $u_{*} \leq u^{*}$, therefore $u^{*}=u_{*}$.

REMARK 3.1. It is easy to see that Theorem 3.1 still holds if $V=V_{1}+V_{2}$ where $\nabla \cdot V_{1}=0$ and $\max _{\mathbb{T}^{n}}\left|V_{2}\right|<1$. 


\section{Conclusions}

G-equations are Hamilton-Jacobi models of front propagation in fluid flows, especially in turbulent combustion. We proved the periodic homogenization of the inviscid incompressible G-equation with convex yet non-coercive Hamiltonians. The effective Hamiltonian is convex, coercive (if the mean of $V$ is zero), and has degree one homogeneity. Though the corrector problem may not have an exact solution due to non-coercivity of the Hamiltonian, homogenization suffices with an approximate corrector whose existence is proved based on the local reachability property of the controlled flow trajectories and incompressibility of the flows. In future work, we shall explore homogenization in more general flows, and compare $\bar{H}$ in viscous and inviscid G-equations.

Acknowledgement. The work was partially supported by NSF grants DMS0712881 (JX), DMS-0848378 and DMS-0901460 (YY).

\section{REFERENCES}

[1] M. Abel, M. Cencini, D. Vergni and A. Vulpiani, Front speed enhancement in cellular flows, Chaos, 12(2), 481-488, 2002.

[2] O. Alvarez and M. Bardi, Ergodic problems in differential games, in Advances in Dynamic Game Theory, S. Jorgensen, M. Quincampoix, and T.L. Vincent (eds.), Ann. Internat. Soc. Dynam. Games, Birkhauser, Boston, 9, 131-152, 2007.

[3] M. Arisawa, Ergodic problem for the Hamilton-Jacobi-Bellman equation, I. Existence of the ergodic attractor. Ann. Inst. H. Poincar Anal. Non Linaire, 14(4), 415-438, 1997.

[4] M. Arisawa, Ergodic problem for the Hamilton-Jacobi-Bellman equation, II. Ann. Inst. H. Poincar Anal. Non Linaire, 15(1), 1-24, 1998.

[5] M. Arisawa and P.L. Lions, On ergodic stochastic control, Commun. Part. Differ. Equs., 23(1112), 2187-2217, 1998

[6] M. Bardi, On differential games with long-time-average cost, Advances in dynamic games and their applications, Ann. Internat. Soc. Dynam. Games, Birkhuser Boston, Inc., Boston, MA, 10, 3-18, 2009.

[7] G. Barles, Some homogenization results for non-coercive Hamilton-Jacobi equations, Calculus of Variations and Part. Differ. Equs., 30(4), 449-466, 2007.

[8] G. Barles, S. Biton, M. Bourgoing and O. Ley, Uniqueness results for quasilinear parabolic equations through viscosity solutions methods, Calculus of Variations, 18, 159-179, 2003.

[9] G. Barles and B. Perthame, Exit time problems in optimal control and vanishing viscosity method, SIAM J. Control Optim., 26(5), 1133-1148, 1988.

[10] H. Berestycki, The influence of advection on the propagation of fronts in reaction-diffusion equations, in Proceedings of the NATO ASI Conference, Cargese, France, H. Berestycki and Y. Pomeau (eds.), Kluwer, Dordrecht, the Netherlands, 2003.

[11] P. Cardaliaguet, Ergodicity of Hamilton-Jacobi equations with a non coercive non convex Hamiltonian in $\mathbb{R}^{2} \backslash \mathbb{Z}^{2}$, Ann Inst. H. Poincaré, Analyse Non Lineaire, to appear.

[12] P. Cardaliaguet, J. Nolen and P.E. Souganidis, Homogenization and Enhancement for the Gequation, Arch. Rat. Mech. Anal., to appear.

[13] P. Clavin and F.A. Williams, Theory of premixed-flame propagation in large-scale turbulence, J. Fluid Mech., 90, 598-604, 1979.

[14] P. Constantin, A. Kiselev, A. Oberman and L. Ryzhik, Bulk burning rate in passive-reactive diffusion, Arch. Ration. Mech. Anal., 154, 53-91, 2000.

[15] M. Crandall, H. Ishii and P.L. Lions, User's guide to viscosity solutions of second order partial differential equations, Bulletin, AMS, 27, 1-67, 1992.

[16] B. Denet, Possible role of temporal correlations in the bending of turbulent flame velocity, Combust Theory Modelling, 3, 585-589, 1999.

[17] P. Embid, A. Majda and P. Souganidis, Comparison of turbulent flame speeds from complete averaging and the G-equation, Phys. Fluids, 7(8), 2052-2060, 1995.

[18] L.C. Evans, The perturbed test function method for viscosity solutions of nonlinear PDEs, Proc. Soc. Edingburgh, section A, 111, 359-375, 1989.

[19] L.C. Evans, Periodic homogenization of certain fully nonlinear partial differential equations, Proc. Royal Soc. Edingburgh, Section A, 120, 245-265, 1992.

[20] W. Fleming and H. Soner, Controlled Markov Processes and Viscosity Solutions, Appl. Math., 
Springer-Verlag, 25, 1993.

[21] H. Ishii, A boundary value problem of the Dirichlet type for Hamilton-Jacobi equations, Ann. Scuola Norm. Sup. Pisa Cl. Sci., (4)16-1, 105-135, 1989.

[22] E. Kosygina, F. Rezakhanlou and S. Varadhan, Stochastic homogenization of Hamilton-JacobiBellman equations, Commun. Pure Appl. Math., 59(10), 1489-1521, 2006.

[23] E. Kosygina and S. Varadhan, Homogenization of Hamilton-Jacobi-Bellman equations with respect to time-space shifts in a stationary ergodic medium, Commun. Pure Appl. Math., 61, 816-847, 2008.

[24] P.L. Lions, G. Papanicolaou and S. Varadhan, Homogenization of Hamilton-Jacobi equations, preprint, 1986.

[25] P.L. Lions and P. Souganidis, Homogenization of viscous Hamilton-Jacobi equations in stationary ergodic media, Commun. Part. Differ. Equs., 30, 335-375, 2005.

[26] Y-Y Liu, J. Xin and Y. Yu, Periodic homogenization of G-equations and viscosity effects, Nonlinearity, to appear.

[27] G. Markstein, Nonsteady Flame Propagation, Pergamon Press, Oxford, 1964.

[28] J. Nolen and J. Xin, Asymptotic spreading of KPP reactive fronts in incompressible space-time random flows, Ann Inst. H. Poincaré, Analyse Non Lineaire, 26(3), 815-839, 2009.

[29] J. Nolen, J. Xin and Y. Yu, Bounds on Front Speeds for Inviscid and Viscous G-equations, Meth. Appl. Anal., to appear.

[30] S. Osher, R. Fedkiw, Level Set Methods and Dynamic Implicit Surfaces, Appl. Math. Sci., Springer, New York, 153, 2003.

[31] N. Peters, A spectral closure for premixed turbulent combustion in the flamelet regime, J. Fluid Mech., 242, 611-629, 1992.

[32] N. Peters, Turbulent Combustion, Cambridge University Press, Cambridge, 2000.

[33] P. Ronney, B. Haslam and N. Rhys, Front propogation rates in randomly stirred media, Phys. Rev. Lett., 74, 3804-3807, 1995.

[34] F. Rezakhanlou and J. Tarver, Homogenization for Stochastic Hamilton-Jacobi Equations, Arch. Rational Mech. Anal., 151, 277-309, 2000

[35] F. Rezakhanlou, Central Limit Theorem for Stochastic Hamilton-Jacobi Eqautions, Commun. Math. Phys., 211, 413-438, 2000.

[36] G. Sivashinsky, Cascade-renormalization theory of turbulent flame speed, Combust. Sci. Tech., $62,77-96,1988$

[37] P. Souganidis, Stochastic homogenization of Hamilton-Jacobi equations and some applications, Asymptotic Analysis 20, 1-11, 1999

[38] R. Schwab, Stochastic Homogenization of Hamilton-Jacobi Equations in Stationary Ergodic Spatial-Temporal Media, Indiana Univ Math J., to appear.

[39] F. Williams, Combustion Theory, Benjamin Cummings, Menlo Park, CA, second edition, 1985.

[40] J. Xin, Front propagation in heterogeneous media, SIAM Review, 42, 161-230, 2000.

[41] J. Xin, An Introduction to Fronts in Random Media, Surveys and Tutorials, Appl. Math. Sci., Springer, 5, 2009.

[42] V. Yakhot, Propagation velocity of premixed turbulent flames, Combust. Sci. Tech., 60, 191241, 1988. 